\title{
New germline mutations in the hypervariable minisatellite CEB I in the parents of children with leukaemia
}

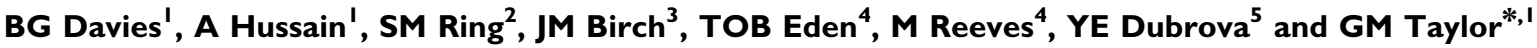 \\ 'Cancer Immunogenetics Laboratory, Division of Human Development, University of Manchester, Manchester, UK; ${ }^{2}$ ALSPAC DNA and Cell Line Bank, \\ University of Bristol, Bristol, UK; ${ }^{3}$ CRUK Paediatric and Familial Cancer Study Group, University of Manchester, Manchester, UK; ${ }^{4}$ Academic Unit of \\ Paediatric Oncology, Division of Human Development, University of Manchester, Manchester, UK; ${ }^{5}$ Department of Genetics, University of Leicester, \\ Leicester, UK
}

\begin{abstract}
Gardner and co-workers advanced the hypothesis that the Seascale leukaemia cluster could have been caused by new mutations in germ cells, induced by paternal preconceptional irradiation (PPI) exposure at the Sellafield nuclear installation. Since evidence has shown that PPI can increase the de novo germline mutation rate in hypervariable minisatellite loci, we investigated the hypothesis that sporadic childhood leukaemia might be associated with an increased parental germline minisatellite mutation rate. To test this hypothesis, we compared de novo germline mutation rates in the hypervariable minisatellite locus, CEBI, in family trios (both parents and their child) of children with leukaemia $(n=135)$ compared with unaffected control families $(n=124)$. The majority of case and control germline mutations were paternal (94\%); the mean paternal germline mutation rates of children with leukaemia (0.083) and control children (0.156) were not significantly different (odds ratio, 95\% confidence interval: 0.50, 0.23-1.08; $P=0.1 \mathrm{I}$ ). There were no significant differences in case and control parental allele sizes, case and control germline mutation progenitor allele sizes (2.74 vs $2.54 \mathrm{~kb} ; P=0.56)$, case and control mutant allele sizes $(2.7 \mathrm{I}$ vs $2.67 \mathrm{~kb} ; P=0.90)$, mutant allele size changes $(0.13 \mathrm{vs} 0.26 \mathrm{~kb} ; P=0.10)$, or mutational spectra. Within the limitation of the number of families available for study, we conclude that childhood leukaemia is unlikely to be associated with increased germline minisatellite instability.

British Journal of Cancer (2007) 96, 1265-127I. doi:I0.1038/sj.bjc.6603706 www.bjcancer.com
\end{abstract}

Published online 27 March 2007

(c) 2007 Cancer Research UK

Keywords: childhood leukaemia; minisatellite; germline; mutation

Acute leukaemia accounts for about $30 \%$ of childhood malignancies in the UK, with an average incidence rate of $4 / 100000 /$ year (Little, 1999; Stiller, 2004). Childhood leukaemia is thought to be initiated during early development by unrepaired chromosome breaks in a haemopoietic progenitor exposed to environmental carcinogens (Greaves, 1997, 1999). However, epidemiological studies have failed, with one exception, to identify decisively these carcinogens. This exception involves exposure of the unborn child to X-rays in utero, which is associated with a $\sim 50 \%$ increased risk of childhood leukaemia (Stewart et al, 1956; Stewart and Kneale, 1968; Stewart and Kneale, 1970; Bithell and Stewart, 1975; Doll and Wakeford, 1997; Wakeford and Little, 2003).

The discovery of an unusual cluster of childhood leukaemia cases in the village of Seascale, close to the Sellafield nuclear site in Cumbria, UK, raised concerns about fetal and neonatal exposure to environmental ionising radiation (IR; reviewed in Beral, Roman and Bobrow, 1993). To identify the cause of the Seascale cluster more precisely, a case - control study of children born in the village compared with those born elsewhere was carried out by Gardner et al (1990). The findings of this study were interpreted as

\footnotetext{
*Correspondence: Dr GM Taylor;

E-mail: gmtaylor@manchester.ac.uk

Received 2 November 2006; revised 28 February 2007; accepted 28 February 2007; published online 27 March 2007
}

suggesting that exposure to IR of fathers working at Sellafield might be leukaemogenic in their offspring, by a mechanism involving IR-induced de novo mutations in sperm during the preconceptional period. This preconceptional irradiation (PPI) hypothesis suggested a new mechanism of leukaemogenesis in humans, and was highly controversial, as there was little evidence from other sources to suggest that childhood leukaemia could be caused by germline mutation. Moreover, fathers' radiation exposure was considered to be insufficient to cause the mutation rate required for the number of cases observed (Evans, 1990; Doll et al, 1994; Wakeford et al, 1994). Additionally, there was no evidence of a germline effect among the children of Japanese atomic bomb survivors (Kodaira et al, 1995, 2004; Izumi et al, 2003). Furthermore, as radiation damage is randomly distributed across the genome, PPI should have caused an increase in single gene disorders (Doll et al, 1994). Although Parker et al (1999) reported an increased risk of stillbirth in the offspring of men working at Sellafield exposed to IR, this was not confirmed by Doyle et al (2000) in a study of nuclear industry workers.

Subsequent to the Gardner study, Dubrova et al (1996) reported that PPI caused an increased de novo germline mutation rate in certain human minisatellite tandem repeat DNA loci. Owing to the high spontaneous germline mutation rate of some minisatellites ( $>1000 \times$ higher than most protein-coding loci), a PPI effect could be detected in a substantially smaller population than required to 
detect mutations in protein-coding loci (Dubrova et al, 1993, 1996, 1997). Studies of two different populations, one exposed to IR from radionuclide-contaminated land following the Chernobyl accident ( $\sim 0.5 \mathrm{~Gy}$, Dubrova et al, 1996, 1997, 2002a), the other from the fallout of nuclear weapons tests ( $>1 \mathrm{~Sv}$; Dubrova et al, 2002b), revealed an approximate doubling in germline minisatellite mutation rates due to PPI. These and other data suggest that minisatellite mutation rates may be useful biomarkers of germline genetic effects caused by environmental mutagens such as IR (Yauk, 2004; Bouffler et al, 2006). As minisatellites do not appear to be the direct target of mutagen-induced DNA damage (Dubrova et al, 1996, 1997), it is thought that they may act as a marker for general genome sensitivity or instability (Niwa, 2003).

On the basis of the PPI hypothesis for the aetiology of the Seascale leukaemia cluster (Gardner et al, 1990), and increased de novo germline minisatellite mutation because of PPI, we surmised that a proportion of sporadic childhood leukaemias might be associated with an increase in the rate of new germline minisatellite mutations owing to undetected exposures of parental germ cells to mutagens such as radiation. To address this, we compared germline mutation rates in the hypervariable human minisatellite, CEB1 (Vergnaud and Denoeud, 2000) in the families of children with leukaemia $(n=135)$ and control families $(n=124)$, using a sensitive PCR-based minisatellite genotyping assay.

\section{MATERIALS AND METHODS}

\section{Case and control samples}

The case families were a cross-sectional series of children with acute leukaemia $(n=145)$ treated at Royal Manchester Children's Hospital, UK, and their parents $(n=288$; two cases were monozygotic (MZ) twins). Blood samples were obtained from case children at diagnosis and in remission and from their parents. Controls were two series of family trios consisting of unaffected children $(n=124)$ and their parents $(n=248)$. One control series consisted of buccal scrape samples from children $(n=64)$ and blood samples from their parents $(n=128)$, recruited as part of the UK Childhood Cancer Study (2000) in the North West (NW) of England. The second control series consisted of blood samples from children $(n=60)$ obtained as part of the Avon Longitudinal Study of Parents and Children (ALSPAC; Jones et al, 2000) and buccal samples from their parents $(n=120$; Ring et al, 2001). Sample collection and analyses were carried out with the approval of Local Research Ethics Committees.

\section{Genomic DNA extraction}

Genomic DNA (gDNA) was extracted from case and control blood samples using phenol/chloroform or Nucleon BACC2 genomic DNA extraction kits (Amersham Biosciences, Chalfont St Giles, UK). gDNA was extracted from buccal samples using MasterAmp ${ }^{\mathrm{TM}}$ Buccal Swab DNA Extraction Kits (Epicentre Technologies, Madison, USA). DNA concentrations were measured by fluorimetry on a TBS-380 Mini-Fluorometer (Turner BioSystems Inc., Sunnyvale, CA, USA) using a PicoGreen ${ }^{\circledR}$ (Molecular Probes, Paisley, UK) based assay.

\section{CEB1 primer sequences and PCR amplification conditions}

CEB1 alleles were amplified by PCR using the $3^{\prime}$ CEB1 primer P14 ( $5^{\prime}$-ggatcctctcctgtgcctttcct- $\left.3^{\prime}\right)$ described by Buard et al (1998) and a $5^{\prime}$ primer, MAR1 ( $5^{\prime}$-gaattttcagtgagagtcggcc- $\left.3^{\prime}\right)$. MAR1 was designed for this study to avoid single nucleotide polymorphisms (SNPs) flanking the CEB1 minisatellite, using the CEB1 sequence (accession no. AF048727) and SNP information kindly provided by
Dr Jerome Buard (personal communication). Polymerase chain reactions were performed on an MJ Research DNA Engine (Waltham, MA, USA) in $50 \mu \mathrm{l}$ reaction mixtures consisting of $1 \mu \mathrm{l}$ of gDNA (50 ng) and $0.5 \mu \mathrm{l}(2.5$ units) of platinum Taq DNA polymerase high fidelity (Invitrogen, Paisley, UK) added to $48.5 \mu \mathrm{l}$ of $\mathrm{Mg}^{2+}$-titrated PCR master-mix. The PCR master-mix $(19.4 \mathrm{ml})$ was prepared with the following final reagent concentrations: $0.5 \mu \mathrm{M}$ each high-performance liquid chromatography-purified CEB1 primer (Eurogentec, Southampton, UK), $0.7 \mathrm{~mm} \mathrm{MgSO}_{4}$ (50 $\mathrm{mM} \mathrm{MgSO}_{4}$ with platinum Taq DNA polymerase), $0.2 \mathrm{~mm}$ dNTPs (PCR nucleotide mix, Amersham Biosciences) $5 \%(\mathrm{v} / \mathrm{v})$ DMSO (Sigma, , Dorset, UK), $0.2 \mathrm{mg} \mathrm{ml}^{-1}$ bovine serum albumin (ultra pure, non-acetylated; Ambion, Warrington, UK), and $1 \times$ high-fidelity PCR buffer $(50 \times$ high-fidelity PCR buffer with platinum Taq DNA polymerase $\mathrm{HF}$ ). Amplification conditions were $94^{\circ} \mathrm{C}$ for $1 \mathrm{~min} 30 \mathrm{~s}, 30$ cycles at $94^{\circ} \mathrm{C}$ for $15 \mathrm{~s}, 60^{\circ} \mathrm{C}$ for $30 \mathrm{~s}$, $70^{\circ} \mathrm{C}$ for $10 \mathrm{~min}$, and a final extension step of $70^{\circ} \mathrm{C}$ for $15 \mathrm{~min}$.

\section{Electrophoresis}

Polymerase chain reaction products containing CEB1 alleles amplified from the gDNA of family trios (father, mother, and child) were loaded onto a $40-\mathrm{cm}$ long $0.7 \%(\mathrm{w} / \mathrm{v})$ agarose gel (SeaKem LE Agarose, Cambrex BioScience, East Rutherford, NJ, USA). Gels were run at $2 \mathrm{~V} \mathrm{~cm}^{-1}$ in $1 \times \mathrm{TBE}$ buffer (Crystal Buffers, Severn Biotech, Kidderminster, UK) for approximately $23 \mathrm{~h}$, until the $400 \mathrm{bp}$ marker from the DNA ladder $(1 \mathrm{~kb}$ plus DNA Ladder, Invitrogen) was about to run off the gel. Few alleles $<400$ bp were observed, but when present were sized by a shorter gel run. Multiple ladder lanes were included across each gel to enable the image analysis software to calculate the gel retardation factor $\left(R_{\mathrm{f}}\right.$ value) for any region of the gel, allowing more accurate allele sizing. Gels were stained with SYBR gold nucleic acid gel stain (Molecular Probes) in $1 \times$ TBE buffer ( $\mathrm{pH} 8.0$ ), the bands being visualised by UV transillumination on a Gel Doc 2000 instrument (Bio-Rad, Hemel Hempstead, UK). The captured gel image in TIFF file format was set up with an image resolution of 38.4 pixels $\mathrm{cm}^{-1}$.

\section{Identification of $C E B 1$ alleles and mutations}

Allele typing and mutation detection were performed on the gel image using Phoretix ${ }^{\mathrm{TM}}$ 1D Advance software (Nonlinear Dynamics, Newcastle upon Tyne, UK). Offspring bands (alleles) were deemed to be germline mutations if no corresponding alleles were detected in either parent. Band shifts were measured in bps so that allele sizes could be adjusted for variations in $R_{\mathrm{f}}$. This was carried out by placing a simulated band on the gel image at a position $1.5 \mathrm{~mm}$ (corresponding to $\sim 8$ pixels) from the band of interest, in the direction of the progenitor band in the parent. The progenitor band was taken to be the parental allele closest in size to the mutant band (Dubrova et al, 1996). If the size of the putative mutant allele was equal to or larger (for an expansion), or equal to or smaller (for a deletion), than the simulated band (as measured in bp), it was classified as a mutation. All mutations detected were verified by repeat analysis, in which gDNA was re-amplified and run on a second gel.

\section{Verification of parentage}

Biological parentage was verified in case and control families from NW England with suspected germline mutations using three short tandem repeat (STR) markers (VWA, TH01, and FGA) from the FBI combined DNA index system (CODIS) and the minisatellite, $D 1 S 80$, as described in the National Institute of Standards and Technology STR DNA internet database (http://www.cstl.nist.gov/ biotech/strbase/; Ruitberg et al, 2001) and using HLA-DPB1 typing data already available for families (Taylor et al, 2002). Primer sequences for STRs were: VWA $5^{\prime}$-ccctagtggatgataagaataatcagtatg- $3^{\prime}$ 
and $5^{\prime}$-ggacagatgataaatacataggatggatgg- $3^{\prime} ;$ THO1 $5^{\prime}$-attcaaagggtat ctgggctctgg- $3^{\prime}, 5^{\prime}$-gtgggctgaaaagctcccgattat- $3^{\prime}$; and $F G A 5^{\prime}$-attatcca aaagtcaaatgccccatagg- $3^{\prime}, 5^{\prime}$-atcgaaaatatggttattgaagtagctg- $3^{\prime}$. Primer sequences for D1S80 were: $5^{\prime}$-gtcttgttggagatgcacgtgcccttgc- $3^{\prime}$, $5^{\prime}$-gaaactggcctccaaacactgcccgccg-3' (Kasai et al, 1990). Highperformance liquid chromatography-purified primers were obtained from Eurogentec or Applied Biosystems (Warrington, UK), the forward primer for each set being labelled at the $5^{\prime}$-end with VIC (VWA), NED (TH01), or 6-FAM (FGA and D1S80). All four loci were co-amplified and analysed on an ABI-310 genetic analyser (Applied Biosystems). Reagent concentrations in $20 \mu \mathrm{l}$ reaction volumes were $1 \times$ ReddyMix $^{\mathrm{TM}}$ PCR master mix with $2.5 \mathrm{mM} \mathrm{MgCl}_{2}$ (ABgene, Epsom, UK), $0.25 \mu \mathrm{M}$ each primer and $2.5 \mathrm{ng}^{-1} \mathrm{l}^{-1} \mathrm{DNA}$. Polymerase chain reaction amplification conditions were $95^{\circ} \mathrm{C}$ for $2 \mathrm{~min}, 30$ cycles at $95^{\circ} \mathrm{C}$ for $30 \mathrm{~s}, 63^{\circ} \mathrm{C}$ for $30 \mathrm{~s}$, $70^{\circ} \mathrm{C}$ for $2 \mathrm{~min}$, and a final extension step of $70^{\circ} \mathrm{C}$ for $5 \mathrm{~min}$. Parentage was verified in mutation carriers if alleles in at least two of the four tandem-repeat loci were present in parents; DP types were used as an independent check. Parentage of all ALSPAC control family trios was verified using four STR loci (D5S818, D7S820, D13S317, D16S539; http://www.cstl.nist.gov/biotech/ strbase/) by multiplex PCR using GammaSTR kits (Promega, Southampton, UK) as described by Ong et al (2004).

\section{Data analysis}

Case-control CEB1 germline mutation rates were compared by calculating cross-product odds ratios (ORs) and 95\% confidence intervals (CI) using the RERI program from the linkage utility package, LINKUTIL, by the Sheehe method (http://linkage. rockefeller. edu/soft/linkutil/). The $2 \times 2$ programme in LINKUTIL was used to determine $P$ values for case-control differences using Fisher's exact test. Mean case-control parental ages and germline progenitor allele sizes were compared using unpaired $t$-tests by Minitab release 13.30. Case-control parental allele size distributions were compared using the Kolmogorov-Smirnov $\mathrm{Z}$ twoindependent samples test. Statistical power was calculated using nQuery Advisor v2.0 (Statistical Solutions, Cork, Ireland).

\section{RESULTS}

\section{Case-control characteristics}

A total of 145 leukaemia cases from 144 families were originally included in the study. Two cases with B-cell precursor ALL (BCP ALL) were MZ twins and were treated as one case in the analysis. Control families consisted of 124 unaffected children and their parents. Nine case families were excluded from the study; five because of failure to detect $C E B 1$ alleles; four because of nonparentage, leaving 135 informative case families. Table 1 shows the number of informative case children with each leukaemia subtype. $\mathrm{B}$-cell precursor ALL is the predominant subtype (70\%), the

Table I Details of leukaemia case series in this study

\begin{tabular}{lccr}
\hline & \multicolumn{2}{c}{ Number of samples obtained at } & \\
\cline { 2 - 3 } Leukaemia cases & Diagnosis & Remission & Total \\
\hline BCP ALL & 66 & 29 & 95 \\
Pro-B ALL & 6 & 2 & 8 \\
T ALL & 10 & 9 & 19 \\
AML $^{2}$ & 11 & 2 & 13 \\
Total cases $^{2}$ & 93 & 42 & 135 \\
\hline
\end{tabular}

AML, acute myeloid leukaemia; BCP ALL, B-cell precursor ALL; Pro-B ALL, progenitor B-cell ALL; T ALL, T-cell ALL. ${ }^{a}$ One case of chronic myeloid leukaemia and one case of myelodysplastic syndrome.

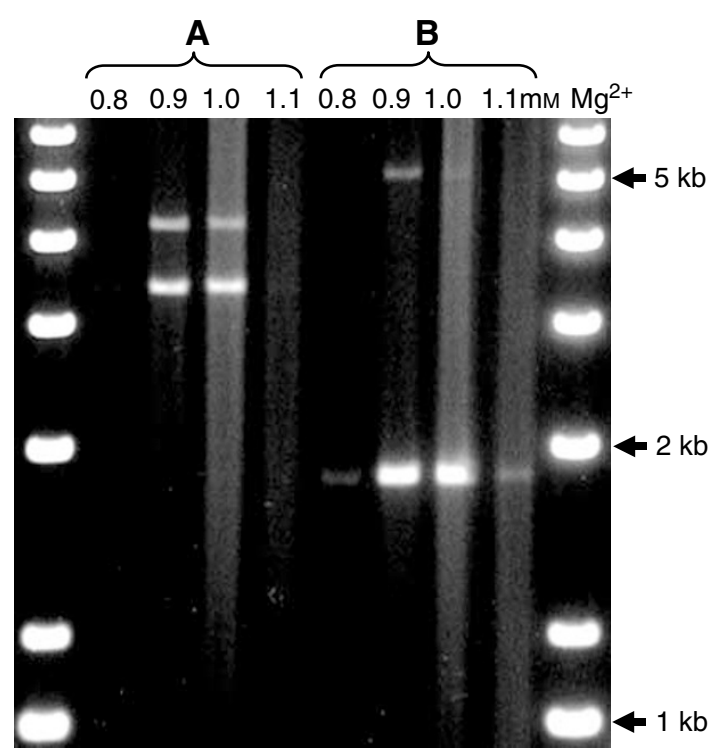

Figure I Effect of $\mathrm{Mg}^{2+}$ concentration on amplification of CEB / alleles in two gDNA samples (A and $\mathbf{B}$ ) by PCR. No alleles were resolved at 0.8 or $1.1 \mathrm{mM} \mathrm{Mg}^{2+}$, whereas alleles were optimally detected at $0.9-1.0 \mathrm{mM}$.

majority $(68 \%)$ of case samples were collected at diagnosis. The mean age of cases at diagnosis of leukaemia was 5 years 5 months (range: $0.2-15.0$ years). The mean paternal age at the birth of case children was $30.4 \pm$ (s.d.) 5.4 years; for the control series were the following, NW: $31.0 \pm 6.3$ years; ALSPAC: $32 \pm 5$ years. Mean maternal ages were: case children $28.5 \pm 5.7$ years; NW controls: $29.1 \pm 4.6$ years; ALSPAC controls: $30 \pm 4.1$ years. There were no significant differences between case and control parental ages $(P>0.05)$

\section{Amplification and genotyping of CEB1 alleles}

CEB1 genotyping was carried out by amplifying gDNA in PCR to levels visible in SYBR gold-stained gels. A very precise $\mathrm{Mg}^{2+}$ concentration (to within $0.1 \mathrm{~mm}$ ) was found to be required for successful amplification and allele discrimination (Figure 1). Using this method, we obtained an allele amplification efficiency of $90 \%$, with no amplification artifacts and consistent results in repeat sample analysis.

\section{Case-control germline mutation rate comparison}

Germline mutations were detected in 11 of 135 case and 20 of 124 control children. Representative examples of mutations (one expansion and one deletion) in two case families are shown in Figure 2. All case mutations were paternal in origin; two of 21 control mutations were maternal. One control but no cases had a double, paternal and maternal, mutation. Table 2 shows that there is no significant difference in parental mutation rates in case (0.041) and control (0.086) families (OR, 95\% CI: 0.47, 0.23-0.98; $P=0.061)$. Paternal mutation rates in case $(0.083)$ and control families (0.156) were also not significantly different (OR, 95\% CI: $0.50,0.23-1.08 ; P=0.11$ ). Analysis of mutation rates in relation to leukaemia subtype (Table 3 ) shows no significant case-control differences, though the small number of pro B and T ALL and acute myeloid leukaemia (AML) suggests that these results should be treated with caution. The subtype with the highest mutation rate was T ALL (0.111), but this was not significantly different from controls (OR, 95\% CI: $0.8,0.22-3.00 ; P=0.93$ ). The mean age of case fathers with a germline mutation (29.9 years) was not significantly different from NW control fathers with a mutation 


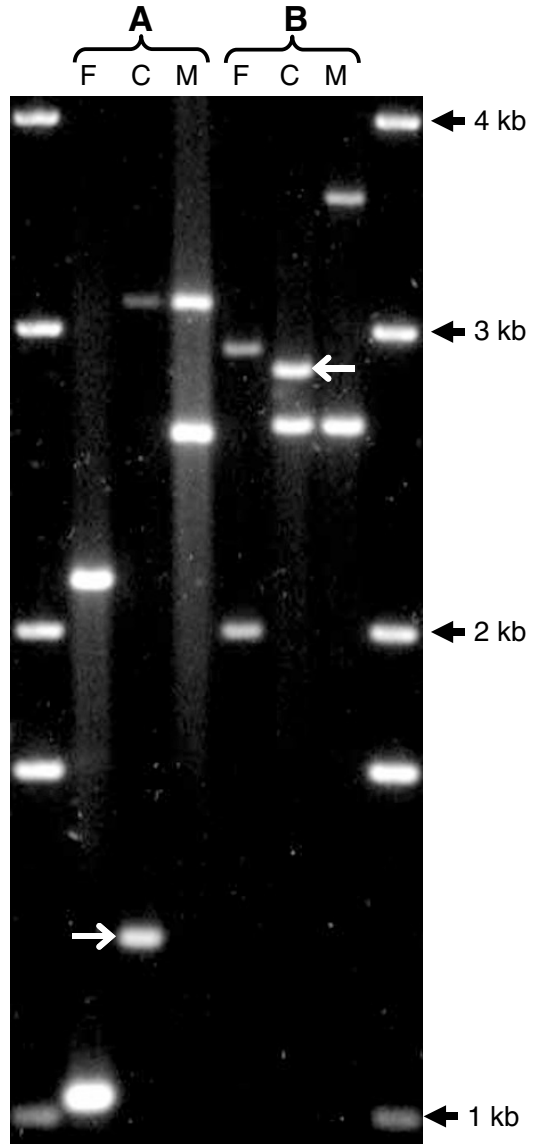

Figure 2 Detection of de novo germline CEB / mutations in two family trios (A and $\mathbf{B}$ ) following PCR and gel electrophoresis. Mutant alleles are indicated by white arrows. Family $\mathrm{A}$ has a paternal mutation corresponding to a seven repeat expansion. Family $B$ has a paternal mutation corresponding to a one repeat deletion. To ensure accurate band comparison between adjacent lanes samples were loaded in the order father $(F)$, child $(C)$, mother $(M)$.
(33.9 years; $P=0.27$ ). The mean age of case fathers whose children had a germline mutation (29.9 years) was not significantly different from fathers of case children who had no mutation (30.4 years; $P=0.81)$. The mean germline mutation rate in diagnostic samples (0.039) was not significantly different from remission samples (0.046; $P>0.05)$. The $C E B 1$ allele size distributions (Figure 3$)$ in case parents (mean allele size: $2865 \mathrm{bp} ; 72$ repeats) was not significantly different from control parents (mean allele size: 2360 bp; 59 repeats; $P>0.05$ ).

\section{Germline mutation spectrum}

In cases, there were seven (64\%) mutant alleles with an increased and four with a decreased number of repeats, whereas in the controls, $15(71 \%)$ mutants increased and six decreased in size. The size of the mutational events was similar in the cases and

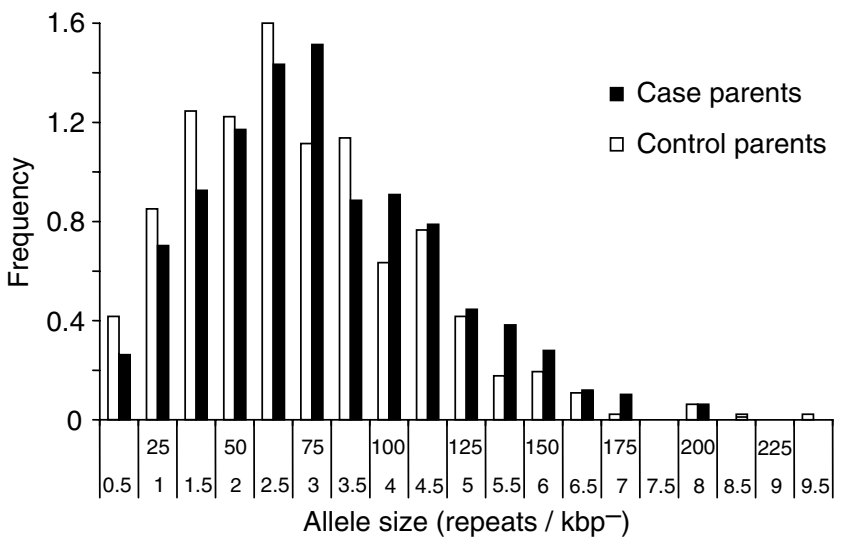

Figure 3 Size distribution of CEB/ alleles in the parents of case and control children (number of alleles: case, $n=496$; control, $n=457$ ). DNA fragments were grouped into $0.5 \mathrm{~kb}$ intervals from 0.5 to $9.5 \mathrm{~kb}$, with the number of repeat units shown, where one consensus repeat $=40 \mathrm{bp}$ ). Differences between case and control parent allele distributions were not significant in the Kolmogorov-Smirnov two-sample test $(P>0.05)$.

Table 2 Germline CEB / mutation rate in parents of childhood leukaemia cases and controls

\begin{tabular}{|c|c|c|c|c|c|}
\hline Comparison & Cases & Controls & OR & $95 \% \mathrm{Cl}$ & Two-sided P* \\
\hline Informative offspring (n) & 136 & 124 & & & \\
\hline Paternal alleles $(n)$ & 132 & 122 & & & \\
\hline Paternal germline mutations & 11 & 19 & & & \\
\hline Maternal germline mutations & 0 & 2 & & & \\
\hline Paternal mutation rate & $0.083(8.3 \%)$ & $0.156(15.6 \%)$ & 0.50 & $0.23-1.08$ & 0.11 \\
\hline
\end{tabular}

$\mathrm{Cl}$, confidence interval; $n$, number; OR, odds ratio. *Two-sided $P$ value in Fisher's exact test.

Table 3 Paternal CEB / germline mutation rate in relation to childhood leukaemia subtype

\begin{tabular}{|c|c|c|c|c|c|c|}
\hline Leukaemia subtype & Paternal alleles & Paternal mutations & Paternal mutation rate & OR & $95 \% \mathrm{Cl}$ & Two-sided $\mathbf{P}$ \\
\hline Pro-B ALL & 8 & 0 & 0 & 0.31 & $0.04-2.60$ & 0.54 \\
\hline T ALL & 18 & 2 & $0.111(11.1 \%)$ & 0.80 & $0.22-0.46$ & 0.93 \\
\hline Total cases & 132 & 11 & $0.083(8.3 \%)$ & 0.50 & $0.23-1.08$ & 0.11 \\
\hline Controls & 122 & 19 & $0.156(15.6 \%)$ & & & \\
\hline
\end{tabular}

AML, acute myeloid leukaemia; BCP ALL; B-cell precursor ALL; Pro-B ALL, progenitor B-cell ALL; T ALL, T-cell ALL. aLeukaemia subtype in relation to paternal allele mutation rate. 


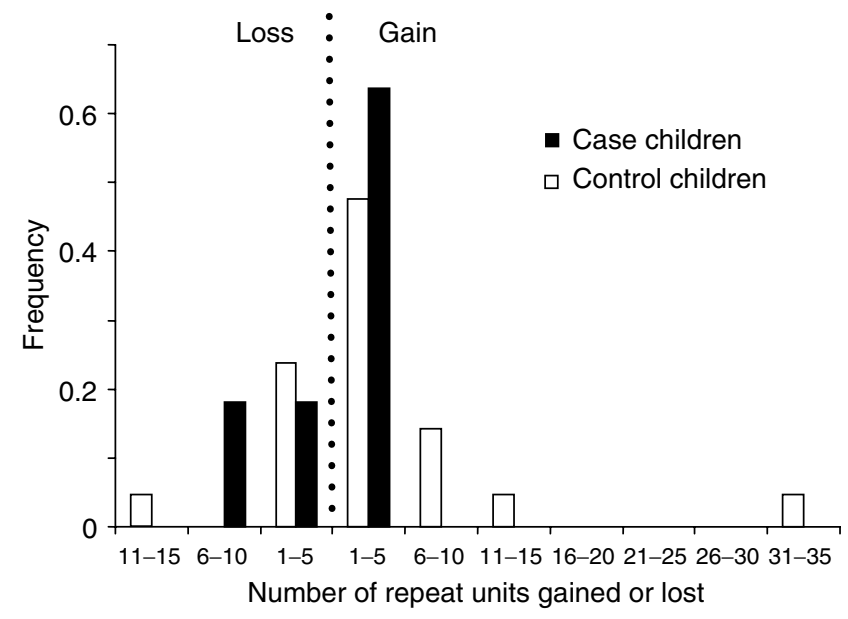

Figure 4 Frequency distribution of germline CEBI mutations in case and control children by size and polarity, with mutations grouped into repeat unit changes of up to five units. In leukaemia case children, all gains are I-5 repeats and losses $1-10$ repeats, whereas in control children gains are $1-35$ repeats and losses $1-15$ repeats.

controls. The mean mutant allele size in cases $(2.71 \mathrm{~kb})$ was not significantly different from controls $(2.67 \mathrm{~kb} ; P<0.90)$. There was no difference between mean progenitor allele size in cases $(2.74 \mathrm{~kb})$ and controls $(2.54 \mathrm{~kb} ; P=0.56)$. Most mutations $(75 \%)$ involved the gain or loss of $1-5$ repeat units (Figure 4 ), but there was no significant difference in the size change in cases $(0.13 \mathrm{~kb})$ and controls $(0.25 \mathrm{~kb} ; P=0.10)$. The largest control mutation was an expansion of 32 repeat units (progenitor allele size: $2.09 \mathrm{~kb}$ ); the largest case mutation was a deletion of nine repeats (progenitor allele size: $2.55 \mathrm{~kb}$ ) in a child with BCP ALL.

\section{DISCUSSION}

This study was designed to address the hypothesis that sporadic childhood leukaemia is associated with an increased rate of de novo germline mutation in the hypervariable minisatellite, CEB1. The results show, with some qualifications, that there is no association. The hypothesis is based on the notion that exposure of parental germ cells to mutagenic agents such as IR can lead to an increased risk of childhood leukaemia, the PPI hypothesis (Gardner et al, 1990). Here, we used minisatellite mutation as a biomarker of de novo germline leukaemogenic damage. We found no evidence for quantitative (germline mutation rate) or qualitative (mutation spectrum) differences in CEB1 minisatellite mutations between case and control families. As far as we are aware no previous study of this type has been reported in childhood leukaemia.

Unlike other studies of germline minisatellite mutation rate in relation to parental IR exposure (reviewed by Bouffler et al, 2006), no parental radiation dosimetry data were available for our leukaemia cases. Accurate environmental radiation and mutagen exposure data are extremely difficult to collect in a nonoccupational setting, but it is safe to assume in these cases that environmental radiation exposures were extremely low. The catchment area for our sporadic cases covers an area of NW England south of Cumbria, where levels of occupational or environmental radiation are far below those to which the Sellafield workers were exposed. As CEB1 exhibits high rates of spontaneous and induced mutation, our study should have revealed evidence of undetected parental mutagen exposure associated with childhood leukaemia. From our prior hypothesis of an increased case de novo germline mutation rate, our study of 135 informative case and 124 control families has $90 \%$ power to detect a significant doubling (one-sided, $P=0.05$ ) of the control paternal mutation rate in cases (i.e., from 0.156 in controls to 0.312 in cases). Although we analysed only one minisatellite locus, in contrast to others using up to eight loci (reviewed by Bouffler et al, 2006), it is worth noting that significance in multiple locus tests requires correction for the number of loci tested, whereas our result does not.

Germline minisatellite mutation analysis has routinely been carried out using Southern blotting and probing of PCR-amplified gDNA (Dubrova et al, 1996; Stead and Jeffreys, 2000). However, this is expensive on gDNA, leading to difficulties in testing children with haemopoietic malignancies where numbers of peripheral blood leucocytes may be reduced by disease or therapy. Using a PCR-based genotyping assay that directly visualised CEB1 alleles on long-read agarose gels using a UV transilluminator, direct analysis of PCR products has provided a robust alternative, allowing fine resolution and sizing of mutations. Careful titration of $\mathrm{Mg}^{2}$ gave reproducible amplification of CEB1 alleles to visible levels, which we found useful for the genotyping of other PCR-able minisatellite loci such as B6.7 (Tamaki et al, 1999). Several other hypervariable minisatellites (Vergnaud and Denoeud, 2000) should be amenable to analysis using this PCR-genotyping assay.

The majority of de novo germline mutations detected in the case and control series in the present study (30/32) were paternal in origin, a result previously reported for CEB1 (Vergnaud et al, 1991). We examined the size change and polarity of mutational events (increase or decrease in allele size), but found no difference between the case and control series. In both groups, allele size expansions were more prevalent than deletions (with an overall ratio of 2.2:1 in favour of expansion) and a large proportion of mutations involved the gain or loss of only $1-3$ repeat units (73 cases and $62 \%$ controls). Small mutations such as these are reminiscent of the stepwise model for microsatellite expansion (Nag, 2003) though the evidence suggests that virtually all CEB1 germline mutations are initiated by recombinogenic events occurring during gametogenesis (Buard et al, 1998, 2000) and not as a result of DNA replication slippage.

In view of the tendency of some single gene disorders to increase in frequency with paternal age, we compared paternal ages at the birth of case and control children with germline mutations. There was no evidence that fathers transmitting germline mutations were significantly older than those who did not. Although mean allele sizes differed slightly between cases and controls (72 and 59 repeats, respectively), there was no significant difference between the allele size distributions suggesting that paternal allele size is not a factor in the risk of leukaemia. This was confirmed by the finding that the sizes of the germline mutation progenitor alleles in cases and controls were not significantly different. The analysis of $C E B 1$ mutation rate in relation to leukaemia subtype was limited by the small number of cases with progenitor B-cell ALL, T ALL, and AML. However, our preliminary finding suggests that none of the leukaemia subtypes had a mutation rate that was higher than the controls.

The type of sample used in the CEB1 genotyping was a potential confounder. We collected $69 \%$ of case samples at diagnosis and $31 \%$ in remission. The diagnostic samples contained varying percentages of leukaemic blasts $(1-96 \%)$, which could have influenced the result if the blasts contained CEB1 somatic mutations. However, comparison of the mutation frequency in the diagnostic (seven mutations in 93 children (7.5\%)) and remission samples (four mutations in 42 children (9.5\%)) suggests little difference. In seven case families, where paired diagnostic and remission samples were available for analysis, we found no differences in CEB1 alleles in the paired samples, even though three of the diagnostic samples had $>73 \%$ blasts. In the control families, buccal instead of blood samples were obtained from children in the NW series and from parents in the ALSPAC series. The overall germline mutation rate in the two series was little 
different (0.0833 (NW) and 0.0877 (ALSPAC)), confirming preliminary studies showing that the genotypes of buccal samples do not differ from blood. The CEB1 somatic mutation rate is known to be very low (Vergnaud et al, 1991; Buard et al, 1998; 2000). Buard et al (2000) reported that the CEB1 somatic mutation rate in blood was $1.8 \times 10^{-4}$ as compared with a $\sim 6 \%$ germline mutation rate in the same donor.

In summary, our results show, within the limitations of the numbers of case and control families available for study, no evidence of an increased rate of de novo CEB1 germline minisatellite mutation in the parents of children with sporadic leukaemia. Our provisional conclusion is that new germline minisatellite mutations, used as a surrogate measure of parental mutagen exposure on germ cell stability, are unlikely to be a major factor in the aetiology of childhood leukaemia. Although our data do not rule out the possibility that prezygotic mutagenesis could account for rarer subtypes of childhood leukaemia, approaches to this question based on population case-control comparisons are unlikely to have sufficient power to provide a definitive result. The report by Dickinson and Parker (2002) confirming the original findings of Gardner et al (1990) of a statistical association between a father's radiation dose at Sellafield and the child's risk of

\section{REFERENCES}

Beral V, Roman E, Bobrow M (1993) Childhood Cancer And Nuclear Installations. London, UK: BMJ Publishing Group

Bithell JF, Stewart AM (1975) Pre-natal irradiation and childhood malignancy: a review of British data from the Oxford survey. $\mathrm{Br} J$ Cancer 31: 271-287

Bouffler SD, Bridges BA, Cooper DN, Dubrova Y, McMillan TJ, Thacker J, Wright EG, Waters R (2006) Assessing radiation-associated mutation risk to the germline: repetitive DNA sequences as mutational targets and biomarkers. Radiat Res 165: 249-268

Buard J, Bourdet A, Yardley J, Dubrova Y, Jeffreys AJ (1998) Influences of array size and homogeneity on minisatellite mutation. EMBO J 17: $3495-3502$

Buard J, Collick A, Brown J, Jeffreys AJ (2000) Somatic versus germline mutation processes at minisatellite CEB1 (D2S90) in humans and transgenic mice. Genomics 65: 95-103

Dickinson HO, Parker L (2002) Leukaemia and non-Hodgkin's lymphoma in children of male Sellafield radiation workers. Int J Cancer 99: 437 - 444

Doll R, Evans HJ, Darby SC (1994) Paternal exposure not to blame. Nature 367: $678-680$

Doll R, Wakeford R (1997) Risk of childhood cancer from fetal irradiation. Br J Radiol 70: 130 - 139

Doyle P, Maconochie N, Roman E, Davies G, Smith PG, Beral V (2000) Fetal death and congenital malformation in babies born to nuclear industry employees: report from the nuclear industry family study. Lancet 356: $1293-1299$

Dubrova YE, Bersimbaev RI, Djansugurova LB, Tankimanova MK, Mamyrbaeva ZZ, Mustonen R, Lindholm C, Hulten M, Salomaa S (2002b) Nuclear weapons tests and human germline mutation rate. Science 295: 1037

Dubrova YE, Grant G, Chumak AA, Stezhka VA, Karakasian AN (2002a) Elevated minisatellite mutation rate in the post-Chernobyl families from Ukraine. Am J Hum Genet 71: 801-809

Dubrova YE, Jeffreys AJ, Malashenko AM (1993) Mouse minisatellite mutations induced by ionizing radiation. Nat Genetics 5: 92-94

Dubrova YE, Nesterov VN, Krouchinsky NG, Ostapenko VA, Neumann R, Neil DL, Jeffreys AJ (1996) Human minisatellite mutation rate after the Chernobyl accident. Nature 380: 683-686

Dubrova YE, Nesterov VN, Krouchinsky NG, Ostapenko VA, Vergnaud G, Giraudeau F, Buard J, Jeffreys AJ (1997) Further evidence for elevated human minisatellite mutation rate in Belarus eight years after the Chernobyl accident. Mut Res 381: 267-278

Evans HJ (1990) Ionising radiations from nuclear establishments and childhood leukaemias-an enigma. BioEssays 12: $541-549$

Gardner MJ, Snee MP, Hall AJ, Powell CA, Downes S, Terrell JD (1990) Results of case-control study of leukaemia and lymphoma among young people near Sellafield nuclear plant in West Cumbria. BMJ 300: 423-429 developing leukaemia suggests that future studies of germline minisatellite mutations in childhood leukaemia should focus specifically on case clusters. Thus, the collection of DNA from family trios in such clusters (Steinberg et al, 2006) has a high priority.

\section{ACKNOWLEDGEMENTS}

This project was carried out with funding provided by the Department of Health, UK (contract RRX81), to Dr GM Taylor. We very gratefully acknowledge receipt of specimens from the patients of the late Dr RF Stevens and from Dr AM Will during the course of this study. We thank Cancer Research (UK) for supporting JM Birch and TOB Eden, and the Medical Research Council and the Paediatric Oncology Fund Royal Manchester Children's Hospital for funding M Reeves. We also thank Ruth Barber (Department of Genetics, University of Leicester) for technical advice and Jerome Buard (Institut de Génétique Humaine, Montpellier, France) for kindly providing CEB1 sequence information.
Greaves MF (1997) Aetiology of acute leukaemia. Lancet 349: 344-349 Greaves M (1999) Molecular genetics, natural history and the demise of childhood leukaemia. Eur J Cancer 35: 1941 - 1953

Izumi S, Koyama K, Soda M, Suyama A (2003) Cancer incidence in children and young adults did not increase relative to parental exposure to atomic bombs. Br J Cancer 89: 1709-1713

Jones RW, Ring S, Tyfield L, Hamvas R, Simmons H, Pembrey M, Golding J, the ALSPAC Study Team (2000) A new human genetics resource: a DNA bank established as part of the Avon Longitudinal Study of Pregnancy and Childhood (ALSPAC). Eur J Human Genetics 8: 653-660

Kasai K, Nakamura Y, White R (1990) Amplification of a variable number of tandem repeats (VNTR) locus (pMCT118) by the polymerase chain reaction (PCR) and its application to forensic science. J Forensic Sci 35: $1196-1200$

Kodaira M, Satoh C, Hiyama K, Toyama K (1995) Lack of effects of atomic bomb radiation on genetic instability of tandem-repetitive elements in human germ cells. Am J Hum Genet 57: 1275-1283

Kodaira M, Izumi S, Takahashi N, Nakamura N (2004) No evidence of radiation effect on mutation rates at hypervariable minisatellite loci in the germ cells of atomic bomb survivors. Radiat Res 162: 350-356

Little J (1999) Epidemiology of Childhood Cancer. Lyon, France: International Agency for Research on Cancer

Nag DK (2003) Trinucleotide repeat expansions: timing is everything. Trends Mol Med 9: 455-457

Niwa O (2003) Induced genomic instability in irradiated germ cells and in the offspring; reconciling discrepancies among the human and animal studies. Oncogene 22: $7078-7086$

Ong KK, Petry CJ, Barratt BJ, Ring S, Cordell HJ, Wingate DL, The Avon Longitudinal Study of Pregnancy and Childhood Study Team Pembrey ME, Todd JA, Dunger DB (2004) Maternal-fetal interactions and birth order influence insulin variable number of tandem repeats allele class associations with head size at birth and childhood weight gain. Diabetes 53: $1128-1133$

Parker L, Pearce MS, Dickinson HO, Aitkin M, Craft AW (1999) Stillbirths among offspring of male radiation workers at Sellafield nuclear reprocessing plant. Lancet 354: 1407-1414

Ring SM, Spanakis E, Beavan T, Barratt B, Jones R, Day I, Pembrey M, Tyfield L, The ALSPAC Study Team (2001) Postal collection of buccal wash samples for DNA: a successful method for expansion of the ALSPAC DNA resource. Eur I Human Genetics 9: 262

Ruitberg CM, Reeder DJ, Butler JM (2001) STRBase: a short tandem repeat DNA database for the human identity testing community. Nucleic Acids Res 29: $320-322$

Stead JDH, Jeffreys AJ (2000) Allele diversity and germline mutation at the insulin minisatellite. Hum Mol Genet 9: 713-723 
Steinberg KK, Relling MV, Gallagher ML, Greene CN, Rubin CS, French D, Holmes AK, Carroll WL, Koontz DA, Sampson EJ, Satten GA (2006) Genetic studies of a cluster of acute lymphoblastic leukemia cases in Churchill County. Nevada. Environ Health Persp in press (doi:10:1289/ ehp.9025)

Stewart A, Kneale GW (1968) Changes in the cancer risk associated with obstetric radiography. Lancet 291: 104-107

Stewart AM, Kneale GW (1970) Age-distribution of cancers caused by obstetric X-rays and their relevance to cancer latent periods. Lancet 296: 4-8

Stewart AM, Webb J, Giles D, Hewitt D (1956) Malignant disease in childhood and diagnostic irradiation in utero. Lancet 268: 447

Stiller CA (2004) Epidemiology and genetics of childhood cancer. Oncogene 23: $6429-6444$

Tamaki K, May CA, Dubrova YE, Jeffreys AJ (1999) Extremely complex repeat shuffling during germline mutation at human minisatellite B6.7. Hum Mol Genet 8: 879-888

Taylor GM, Dearden S, Ravetto P, Ayres M, Watson P, Hussain A, Greaves $M$, Alexander F, Eden OB, UKCCS Investigators (2002) Genetic susceptibility to childhood common acute paediatric lymphoblastic leukaemia is associated with polymorphic peptide-binding pocket profiles in HLA-DPB1*0201. Human Mol Genet 11: 1585-1597

UK Childhood Cancer Study Investigators (2000) The United Kingdom Childhood Cancer Study: objectives, materials and methods. Br J Cancer 82: $1073-1102$

Vergnaud G, Denoeud F (2000) Minisatellites: mutability and genome architecture. Genome Res 10: 899-907

Vergnaud G, Mariat D, Apiou F, Aurias A, Lathrop M, Lauthier V (1991) The use of synthetic tandem repeats to isolate new VNTR loci: cloning of a human hypermutable sequence. Genomics 11: 135-144

Wakeford R, Little MP (2003) Risk coefficients for childhood cancer after intrauterine irradiation: a review. Int J Radiat Biol 79: 293-309

Wakeford R, Tawn EJ, McElvenny DM, Binks K, Scott LE, Parker L (1994) The Seascale childhood leukaemia cases - the mutation rates implied by paternal preconceptional radiation doses. J Radiol Prot 14: 17-24

Yauk CL (2004) Advances in the application of germline tandem repeat instability for in situ monitoring. Mut Res 566: 169-182 